This is an author produced version of a paper published in Wien Klinische Wochenschrift. This paper has been peer-reviewed but does not include the final publisher proof-corrections or journal pagination.

Citation for the published paper:

Bennet, Louise and Fraenkel, Carl-Johan and Garpmo, Ulf and Halling, Anders and Ingman, Mikael and Ornstein, Katharina and Stjernberg, Louise and Berglund, Johan.

"Clinical appearance of erythema migrans caused by Borrelia afzelii and Borrelia garinii - effect of the patient's sex"

Wien Klinische Wochenschrift, 2006, Vol: 118, Issue: 17-18, pp. 531-37. http://dx.doi.org/10.1007/s00508-006-0659-1

Access to the published version may require journal subscription. Published with permission from: Springer 


\section{Clinical appearance of erythema migrans caused by Borrelia afzelii and Borrelia garinii - effect of the patient's sex.}

LOUISE BENNET ${ }^{1,2}$, CARL-JOHAN FRAENKEL ${ }^{4}$, ULF GARPMO ${ }^{5}$, ANDERS HALLING $^{2}$, MIKAEL INGMAN ${ }^{6}$, KATHARINA ORNSTEIN $^{6}$, LOUISE STJERNBERG ${ }^{3}$, JOHAN BERGLUND ${ }^{1,2,3}$.

${ }^{1}$ Department of Clinical Sciences, the University Hospital of Malmö, Lund University, ${ }^{2}$ Blekinge Institute of Research and Development, Karlskrona, ${ }^{3}$ Blekinge Institute of Technology, School of Health Science, Karlskrona, ${ }^{4}$ Department of Infectious Diseases, Blekinge County Hospital Karlskrona, ${ }^{5}$ Department of Clinical Microbiology, Kalmar County Hospital, ${ }^{6}$ Department of Clinical and Experimental Infectious Medicine, the University Hospital of Lund, Sweden.

\section{Address of Correspondance}

MD, PhD Louise Bennet, FoU Blekinge, Gamla Infartsvägen 30, 37141 Karlskrona, Sweden. e-mail: louise.bennet@skane.se

Telephone: +46406234900

Fax number: +46455735548 


\section{ABSTRACT}

Aim: The aim in this survey was to study the clinical characteristics of infections caused by Borrelia genospecies in patients with erythema migrans where borrelial origin was confirmed by polymerase chain reaction. The aim was also to study factors influencing the clinical appearance of erythema migrans.

Methods: This study was conducted in southern Sweden from May 2001 to December 2003 on patients 18 years and older attending with erythema migrans at outpatient clinics. All erythema migrans were verified by polymerase chain reaction, photographed and categorized as "annular" or "non-annular" lesions. A logistic regression model was used to analyze relations between the appearance of the erythema migrans (i.e., annular or non-annular) and factors that influenced its clinical appearance.

Results: A total of 118 patients, 54 women (45.8\%) and 64 men (54.2\%), fulfilled the inclusion criteria. Of these patients, $74 \%$ were infected by B. afzelii and $26 \%$ by B. garinii ( $p$ $<0.001)$. A total of $45 \%(38 / 85)$ of the erythema migrans were annular, $46 \%(39 / 85)$ were non-annular and 9.4\% (8/85) were atypical. For men infected by B. afzelii, the odds ratio of developing non-annular erythema migrans was 0.09 (95\% CI: $0.03-0.33)$ in comparison with women with the same infection.

Conclusions: In this prospective study of a large series of erythema migrans, where genospecies were confirmed by polymerase chain reaction, the sex of patients infected with B. afzelii had a strong influence on the appearance of the rash. Patients infected by B. garinii more often had non-annular erythema migrans and a more virulent infection with more individuals presenting with fever, elevated levels of C-reactive protein and seroreactivity in the convalescence sera.

Key words: Lyme borreliosis, erythema migrans, Borrelia garinii, Borrelia afzelii, polymerase chain reaction. 


\section{INTRODUCTION}

Lyme borrelios is caused by tick-borne spirochetes belonging to the genospecies Borrelia burgdorferi sensu lato (s.1.). The most common clinical manifestation is the non-disseminated cutaneous form erythema migrans (EM) occurring at the site of inoculation (1-3). The geographical distribution of Borrelia genospecies differs in the USA and Europe; in the USA all isolates have been members of the genomic group Borrelia burgdorferi sensu stricto (s.s.), whereas in Europe EM is mostly caused by the spirochetes B. afzelii and B. garinii (4-6). In culture-confirmed EM, patients bitten in the USA develop systemic symptoms and seroreactivity more often than European patients with EM caused by B. afzelii (7). Also, European patients infected by B. garinii more frequently present with flu-like symptoms than those infected with B. afzelii $(8,9)$.

EM is typically "annular" with a central clearing or "homogeneous", but atypical lesions may occur $(3,10-12)$. There has been some debate on whether the different predominant patterns reflect different genospecies or the duration of EM at presentation $(8,9,13)$.

There are few reports in Europe on differences in clinical manifestations caused by the various genospecies of Borrelia burgdorferi s.1. The aim of this study was to investigate the clinical characteristics of infections caused by Borrelia genospecies in patients with EM where genospecies were confirmed by polymerase chain reaction (PCR). An additional aim was to describe and categorize EM patterns and the factors influencing their clinical appearance.

\section{METHODS}

\section{Study Area and Population}

This study was part of the multi-centre "EM biopsy study" conducted in southern Sweden from May 2001 to December 2003. The study population included patients seeking care at seven outpatient clinics in the county of Blekinge.

\section{Study Design}

This prospective study included patients 18 years and older seeking care for EM $>5 \mathrm{~cm}$ in diameter where a borrelial origin of EM was verified through a positive PCR analysis.

The patients gave their written informed consent to participate in this study. During their visit to the doctor, each participant answered a questionnaire asking about the tick bite, the 
erythema and clinical symptoms. The erythema, together with an ID number and a plastic ruler measuring the area of the EM, was photographed using a digital camera. Complete blood counts and liver function tests were performed and C-reactive protein (CRP) levels were measured. Titers of IgM and IgG were mwasured according to the manufacturer's protocol (kit 6029,Dako;antigen, B. afzelii; strain DK-1). Skin-punch biopsies were taken from the leading edge of the EM after the administration of local anaesthetics, and the biopsies were analysed with PCR targeting the ospA gene.

All patients were treated with $\mathrm{V}$ penicillin $(\mathrm{pcV}) 1$ gram, 3 times daily for 10 days or doxycycline $200 \mathrm{mg}$ daily for 10 days, according to the recommendations by the Swedish Medical Product Agency (14). After 14-21 days, patients were contacted by a nurse and asked about their clinical symptoms and the clinical appearance of the EM.

\section{Classification of EM}

The pictures of the lesions were classified by three physicians with extensive experience of treating patients with Lyme borreliosis. The lesions were classified into the following predominant patterns:

- Annular erythemas; round to oval, sharply demarked, red to bluish-red lesions with a classic central clearing $(10,11)$. The "bulls eye rash" is a type of annular EM with a darker, central, bluish-red macula, separated from the peripheral ring by normal skin $(11,12)$. [Fig.I]

- Non-annular erythemas, including homogeneous erythemas; homogeneous red sharply demarked lesions $(10,11)$ and central erythemas; dense central, red to bluish-red lesions surrounded by a paler-red peripheral ring (10). [Fig. II].

- Atypical erythemas; lesions and/or pictures of lesions not assignable to any of the above categories, mainly because of artefacts such as the influence of local anesthetics or photos not possible to interpret.

\section{PCR and sequence analysis}

In order to confirm the EM diagnosis and determine the infecting genospecies, Borrelia DNA was amplified using a nested OspA PCR followed by nucleotide sequencing as previously described (15). Briefly, DNA extraction was performed using a DNeasy tissue kit (Qiagen, Valencia, Ca) according to manufacturer's protocol using an elution volume of $50 \mu 1$. The master mix (PCR Core Kit, Roche Diagnostics GmbH, Penzberg, Germany) contained $0.2 \mu \mathrm{M}$ 
of each primer, $0.2 \mu \mathrm{M}$ of each deoxynucleoside triphosphate and 1.25U Taq DNA polymerase. A volume of $5 \mu 1$ and $1 \mu \mathrm{l}$ DNA template was used in the first and second PCR reaction, respectively. The PCR amplification conditions were: 35 cycles of $94^{\circ}$ for $30 \mathrm{~s}, 50^{\circ}$ for $60 \mathrm{~s}$ and $72^{\circ}$ for $60 \mathrm{~s}$. DNA amplicons were visualized by electrophoresis on a $2 \%$ agarose gel stained with ethidium bromide. Positive DNA samples were sequenced using the OspA PCR inner primer pair (15) and an ABI PRISM BigDye Terminator v.3.1 Ready Reaction Cycle Sequencing Kit (ABI, www.appliedbiosystems.com) according to manufacturers protocol. Each strand was analysed in an ABI 3100 Genetic Analyzer (Applied Biosystems) by the Biomolecular Resource Facility at Lund University. The BioEdit software (Tom Hall, Department of Microbiology, North Carolina State University, NC, USA) was used for nucleotide sequence analysis.

\section{Statistics}

Student's $t$-test was used when comparing normally distributed continuous data. The Pearson chi-square test was used when comparing categorical data. The Mann-Whitney U-test or the Kruskal-Wallis test, was used when comparing non-parametric continous data. All $p$-values were two-tailed and $p$-values $<0.05$ were considered statistically significant.

To evaluate which factors influenced lesion appearance, we used a logistic regression model to analyze the relations between clinical appearance (i.e., annular or non-annular EM) and age, sex, genospecies and time (i.e., time from tick bite to diagnosis). "Female sex" and " $B$. afzelii" were set as baseline variables. The associations between the variables studied were appraised by odds ratios estimating the relative risks for non-annular EM.

SPSS version 11.0 (SPSS Corp., Chicago, IL, USA) or Stata, version 8.0 (Stata Corp., TX, USA) were used for statistical analyses.

\section{Ethical Considerations}

The study was approved by the Ethical Committee at Lund University. 


\section{RESULTS}

\section{Distribution of patients by age and sex}

In this study, 118 patients, 54 women (45.8\%) and 64 men (54.2\%), fulfilled the inclusion criteria. The median age was 56.0 years (range 19.0-94.0) where the median age of the women was 59.0 years $(27.0-94.0)$ and that of the men 53.5 years $(19.0-80.0 ; p=0.020)$.

\section{Tick bites}

In total, 92.4\% (109/118) of the patients had noticed a tick bite at the location of a later EM skin lesion. The most common localisations of the erythemas were legs and feet $(63.6 \%$; $75 / 118)$ followed by chest and back $(24.6 \% ; 29 / 118)$, arms $(10.2 \% ; 12 / 118)$ and genitals (1.7\%; 2/118). Most individuals were bitten when gardening or visiting meadows (48.4\%; 44/91). In addition, $26.4 \%$ (24/91) were bitten by the seashore and $25.3 \%(23 / 91)$ in forest. There were no significant differences in the distribution of B. afzelii and B. garinii infections according to tick habitat [Table 1a]. Most individuals were tick bitten in June $(63.5 \% ; 61 / 96)$ [Fig. III]

\section{Clinical appearances of EM}

In $72.0 \%(85 / 118)$, photographs were taken and the lesions categorized. The median size for the involved lesions was $80.0 \mathrm{~cm}^{2}$. Just as many lesions were annular $(44.7 \% ; 38 / 85)$ as nonannular $(45.8 \% ; 39 / 85)$. Of the non-annular lesions, $27.0 \%(23 / 85)$ were homogeneous and $18.8 \%(16 / 85)$ were central erythemas. A total of $9.4 \%(8 / 85)$ were classified as atypical erythemas.

Most lesions caused by B. afzelii were annular, while most caused by B. garinii were nonannular [Table 1a]. There was a male preponderance of annular EM (27/38) and a female preponderance of non-annular EM $(26 / 39 ; p=0.001)$. The EM on the abdominal region and back were not bigger than the EM on the legs, arms or neck $(p=0.607)$ and they did not appear faster than the EM on the extremities and neck $(p=0.134)$. The annular erythemas were significantly bigger than the non-annular erythemas $(p=0.011)$ and the duration from bite to diagnosis was significantly longer for patients with annular EM than for patients with nonannular EM $(p=0.044)$ [Table 1a].

The variables were studied in a logistic model in sequential steps to identify variables affecting the clinical appearance of the EM. The variable "size of the lesion" was not included in the model since this was considered being influenced by time (16). We studied the 
influence of age, sex, genospecies and time (i.e., time from tick bite to diagnosis) on the clinical appearance of the EMs. The variable "time" was excluded from the model after it was found to have no significant influence on appearance. The variables "female sex" and " $B$. afzelii" were set as baseline variables. For men infected by B. afzelii the odds ratio of developing non-annular EM was only 0.09 (95\% CI: 0.03-0.33), whereas for women and men infected by B. garinii, the odds ratios of developing non-annular EM were similar, i.e., 1.74 (95\% CI: $0.29-10.34)$ and 1.98 (95\% CI: 0.34-11.56) respectively. The odds ratio of the interaction factor between patients' sex and genospecies was 12.46 (95\% CI: 0.98 - 158.80) [Table 2].

\section{Clinical aspects and laboratory results according to genospecies and sex of patient}

Characteristics of EM caused by B. afzelii and B. garinii are summarized in Table 1. Significantly more individuals had EM caused by B. afzelii than by B. garinii, i.e., $73.7 \%$ vs. $26.3 \%(p<0.001)$. There were no sex differences with respect to which Borrelia species had caused the EM. Individuals infected by $B$. afzelii were younger than those infected by $B$. garinii $(p=0.005)$. The median number of days from tick bite to initial visit to the doctor was 17.5 days (d) (3.0-97.0 d). Patients with lesions caused by B. garinii had a shorter duration from tick bite to diagnosis than patients with lesions caused by B. afzelii $(p=0.011)$ [Table 1a].

All lesions healed within about a week after starting treatment, but there were no differences in this respect between lesions caused by B. afzelii and those caused by B. garinii or between annular and non-annular lesions [Table 1a]. However, the EM disappeared faster in men than in women; median $7.0 \mathrm{~d}(1.0-21.0 \mathrm{~d})$ compared with median $11.0 \mathrm{~d}(2.0-35.0 \mathrm{~d})(p=0.008)$.

Local symptoms were reported by $24.6 \%$ (29/118) of the patients. The most common systemic symptoms were headache $(27.1 \% ; 32 / 118)$ followed by muscle/joint pain $(14.4 \%$; $17 / 118)$, and chills $(10.1 \% ; 12 / 118)$. Less common were airway symptoms $(8.5 \% ; 10 / 118)$, neurological symptoms $(6.8 \% ; 8 / 118)$, neck stiffness $(3.4 \% ; 4 / 118)$, and photosensitivity $(1.7 \% ; 2 / 118)$. Four patients $(12.9 \% ; 4 / 31)$ infected with B. garinii had temperatures above $38.0^{\circ} \mathrm{C}$, compared with none of the patients infected with B. afzelii $(p=0.003)$. No other significant differences in the frequency of clinical symptoms were found in relation to the sex of the patient or the infecting genospecies [Table $1 \mathrm{~b}$ ] 
Significantly more patients with EM caused by B. garinii had elevated levels of CRP than did patients with EM caused by B. afzelii $(p=0.006)$. There were no differences according to sex or infecting genospecies in complete blood counts or liver tests [Table 1c]

A total of $40.4 \%$ of the acute serology results were positive but there were no significant differences between B. afzelii and B. garinii infections or according to patients' sex. However, after six weeks significantly more women than men were seropositive (women $71.8 \%$, men $41.9 \% ; p=0.013$ ) and more individuals infected by B. garinii were seropositive compared with those infected by B. afzelii (B. garinii $72.7 \%$, B. afzelii $45.0 \%$; $p=0.017$ ) [Table 1c] In total $100 / 118$ were treated with penicillin $\mathrm{V}(\mathrm{pcV})$ and $18 / 118$ with doxycycline, with no significant differences between B. afzelii and B. garinii infections (B. afzelii 72/87, B. garinii $28 / 31)$; however, significantly more men than women were treated with $\mathrm{pcV}(92.2 \%, 59 / 64$ versus $75.9 \%, 41 / 54 ; p=0.014)$. 


\section{DISCUSSION}

In this prospective study of patients from southern Sweden with EM verified by PCR, 74\% of patients were infected by B. afzelii and $26 \%$ by B. garinii. We found an interesting interaction between the sex of the patient and the infecting genospecies, namely that the clinical appearance of the EM depended on whether B. afzelii was infecting a man or a woman. When infected by B. afzelii, men developed annular EM more often and women developed nonannular EM more often.

In an earlier Swedish study (13), the appearance of annular EM was estimated as a function of time, however the influence of a patients' sex and the particular genospecies was not considered in that study. In the present study, we found no correlation between appearance and median time from tick bite to diagnosis. Other studies have indicated that B. garinii mostly causes homogeneous $\operatorname{EM}(8,9)$ and that different genospecies disseminate with varying intensity, resulting in different symptoms $(7,8,10,13,16)$. In the USA, EM is caused solely by B. burgdorferi s.s. and, in comparison with European EM caused by B. afzelii, patients in the USA more often have non-annular EMs that develop faster $(7,8,10,13,16)$. In addition, infections with $B$. burgdorferi s.s. are reported to be more virulent than infections with B. afzelii (7). In our study we found that EM caused by B. garinii developed faster and was more likely to be non-annular. Thus, genospecies' varying virulence and tendency to disseminate could affect lesion appearance. However, we also found that the sex of the patient, by interacting with the infecting genospecies, was of importance. More women than men had detectable antibodies against B. burgdorferi s.l. six weeks after diagnosis and we also found that men's lesions disappeared faster than women's. Altogether different immunological reactions in the two sexes might explain our findings. Most women in our study were postmenopausal with a median age of 59 years old. After menopause the levels of oestrogen in women decrease leading to an altered immune status (17). The "type-1" cellmediated immune response is important in eradicating the spirochete in humans and the "type-2" antibody mediated immune response is important in down-regulating the "type-1" response and the anti-inflammatory process (18). A study of Swedish patients reinfected with LB has indicated that this "type-1" immune response is less intense in postmenopausal women than in men (19).

Patients in the USA are reported as having local and systemic symptoms that disseminate to other organ systems more often than European patients $(7,10,16)$. In the present study the frequency of systemic symptoms was less than reported in the USA, but was similar to other 
European studies $(8,24)$. Concerning genospecies, four individuals with B. garinii infections had fever, compared with none of the patients infected by B. afzelii. Fever has seldom been reported in individuals with lesions caused by $B$. afzelii, whereas in individuals with lesions caused by B. burgdorferi s.s. or B. garinii fever and other systemic symptoms are much more common $(7,8)$. Our results also indicate a faster increase of $B$. garinii lesions and that individuals infected by B. garinii more often had raised levels of CRP. After six weeks, significantly more individuals infected by $B$. garinii were seropositive. Overall, patients infected by B. garinii were more likely to have fever, faster-developing and faster-spreading EM lesions and raised levels of CRP and were more likely to be seropositive, all indicating that infections caused by B. garinii are more virulent than those caused by B. afzelii. Our findings are supported by others (7-9).

In this study individuals infected by $B$. garinii were significantly older than individuals infected by B. afzelii. This interesting finding has also been reported in a Slovenian study (8). We do not have an explanation for this observation. Nevertheless, there is a tendency for $B$. garinii infections to occurre later in the season than B. afzelii infections [Fig. III] and in an epidemiological study conducted in the county of Blekinge, older persons were infected later in the season than younger ones (28). Perhaps spring fed ticks mainly harbour B. afzelii, and autumn fed ticks $B$. garinii, and the main reservoir hosts in the spring are rodents infected by B. afzelii and in the autumn birds mainly infected with B. garinii $(21,22)$ ?

Over a quarter of the EMs in this study were caused by B. garinii. In a previous study in southern Sweden 1994-1997, only 6.1\% of EM were caused by B. garinii (20). The increased frequency of EM caused by B. garinii in the present study might be explained by increasing knowledge and recognition of the varying appearances of EM among physicians in the area, but might also indicate an increasing importance of birds as a tick reservoir in the area. Birds are the main reservoir for B. garinii-infested ticks $(21,22)$ and in $1999,31 \%$ of spirocheteinfected host-seeking ticks in the area contained B. garinii spirochetes (23).

As reported by others a minority of the patients with EM had detectable antibodies against $B$. burgdorferi s.l. at the initial visit, showing that the sensitivity of serology testing is too low to use as a diagnostic tool in clinical practice $(25,26)$.

In our study, almost every patient had noticed a tick bite, in comparison with other studies in which about every second patient noted the vector $(13,24,27-29)$. This is possibly due to increased awareness of Lyme borreliosis in the population as a result of extensive research on 
Lyme borreliosis conducted in the area since 1990, research often followed and reported by the media.

Most of the individuals in our study sustained their tick bites in June, corresponding well with the vacation months and with earlier European data $(24,30)$ Distribution of the tick bites predominantly on the lower limbs agrees with earlier studies in Sweden $(2,13)$.

Ticks prefer deciduous woodlands (31) harbouring hosts such as deer and rodents. However, most tick bites occurred in gardens or meadows, areas more visited by humans than the forest (32) which indicates that human exposure is of greater importance than tick abundance when estimating the risk of a tick bite.

In conclusion, in this prospective study of a large set of EMs where borrelial origin was confirmed by PCR, an interaction between the sex of the patient and the infecting genospecies was found, where the clinical appearance of EM caused by B. afzelii infection was sexdependent. Further, patients infected by B. garinii had more extensive symptoms, indicating a more intense local and systemic inflammatory reaction than in patients infected by B. afzelii.

\section{ACKNOWLEDGEMENTS}

We thank all doctors in the county of Blekinge that contributed to the inclusion of patients in this study. We are grateful to Pia Gunnarsson for data processing and Karin Holmkvist for help with collecting data. 


\section{REFERENCES:}

1. Steere AC (2001) Lyme disease. N Engl J Med 345:115-125.

2. Berglund J, Eitrem R, Ornstein K, Lindberg A, Rignér Å, Elmrud H et al (1995) An epidemiological study of Lyme disease in southern Sweden. N Engl J Med 333:13191324.

3. Åsbrink E (1991) Cutaneous manifestations of Lyme borreliosis; Clinical definitions and differential diagnosis. Scand J Infect Dis Suppl 77:44-50.

4. Van Dam AP, Kuiper H, Vos K, Kramer MD, Widjojokusomo A, de Jongh BM, et al. (1993) Different genospecies of Borrelia burgdorferi are associated with distinct clinical manifestations of Lyme borreliosis. Clin Infect Dis 17:708-717.

5. Balmelli T, Piffaretti JC (1995) Association between different clinical manifestations of Lyme disease and different species of Borrelia burgdorferi sensu lato. Res Microbiol 146:329-340.

6. Anthonissen FM, De Kesel M, Hoet PP, Bigaignon GH (1994) Evidence for the involvement of different genospecies of Borrelia in the clinical outcome of Lyme disease in Belgium. Res Microbiol 145:327-331.

7. Strle F, Nadelman RB, Cimperman J, Nowakowski J, Picken RN, Schwartz I, et al (1999) Comparison of culture-confirmed erythema migrans caused by Borrelia burgdorferi sensu stricto in New York and by Borrelia afzelii in Slovenia. Ann Intern Med 130:32-36.

8. Logar M, Ruzić-Sabljić E, Maraspin V, Lotric-Furlan S, Cimperman J, Jurca T, et al (2004) Comparison of erythema migrans caused by Borrelia afzelii and Borrelia garinii. Infection 32:15-19.

9. Carlsson SA, Granlund H, Jansson C, Nyman D, Wahlberg P (2003) Characteristics of erythema migrans in Borrelia afzelii and Borrelia garinii infections. Scand J Infect Dis 35:(1): 31-33.

10. Smith RP, Schoen RT, Rahn DW, Sikand VK, Nowakowski J, Parenti D, et al (2002) Clinical characteristics and treatment outcome of early Lyme disease in patients with microbiologically confirmed erythema migrans. Ann Intern Med 136: 421-428. 
11. Müllegger RR (2001) Clinical aspects and diagnosis of erythema migrans and Borrelial lymphocytoma. Acta dermatovenerologica Alpina Pannonica et Adriatica (Available from: http://www.mf.uni-lj.si/acta-apa/acta-apa-01-4/acta-apa-01-4.html)

12. EUCALB. European Union Concerted Action on Lyme Borreliosis (1997-2005) Clinical features of erythema migrans 1997-2005 (Available from: http://www.oeghmp.at/eucalb/diagnosis_clinical-features-ds.html.)

13. Åsbrink E, Olsson I (1985) Clinical manifestations of erythema chronicum migrans afzelius in 161 patients. Acta Derm Venereol Suppl (Stockh) 65: 43-52.

14. Läkemedelsverket (Medical Product Agency).1998. Behandling av och profylax mot fästingöverförda infektioner - behandlingsrekommendationer. (Treatment and profylaxis against tick-borne infections - treatment recommendations) Information från Läkemedelsverket (In swedish) 9 (2), ISSN 1101-7104.

15. Ornstein, K., Berglund J, Bergström S, Norrby R, Barbour AG (2002) Three major Lyme Borrelia genospecies (Borrelia burgdorferi sensu stricto, B. afzelii and B. garinii) identified by PCR in cerebrospinal fluid from patients with neuroborreliosis in Sweden. Scand J Infect Dis 34(5): 341-346.

16. Nadelman RB, Nowakowski J, Forseter G, Goldberg NS, Bittker S, Cooper D, et al (1996) The clinical spectrum of early Lyme borreliosis in patients with cultureconfirmed erythema migrans. Am J Med 100:502-506.

17. Olsén NJ, Kovacs WJ (1996) Gonadal steroids and immunity. Endocr Rev. 17:369384.

18. Widhe M (2003) Immune responses in human Lyme borreliosis. Cytokines and IgG subclasses in relation to clinical outcome. Linköping University Medical Dissertations No 778. Linköping, Sweden.

19. Jarefors S, Bennet L, You E, Forsberg P, Ekerfelt K, Berglund J, et al (2006) Lyme borreliosis reinfection: might it be explained by a gender difference in immune response? Immunology 118:224-232.

20. Ornstein K, Berglund J, Nilsson I, Norrby R, Bergstrom S (2001) Characterization of Lyme borreliosis isolates from patients with erythema migrans and neuroborreliosis in southern Sweden. J Clin Microbiol 39(4):1294-1298. 
21. Olsen B, Duffy DC, Jaensson TGT, Gylfe Å, Bonnedahl J, Bergström S (1995) Transhemispheric exchange of Lyme disease spirochetes by seabirds. J Clin Microbiol $33: 3270-3274$.

22. Olsen B, Jaensson TGT, Bergström S (1995) Prevalence of Borrelia burgdorferi sensu lato-infected ticks on migrating birds. Appl Environ Microbiol 61:3082-3087.

23. Fraenkel C.J, Garpmo U, Berglund J (2002) Determination of novel Borrelia genospecies in Swedish Ixodes ricinus ticks. J Clin Microbiol 40(9): 3308-3312.

24. Strle F, Videčnik J, Zorman P, Cimperman J, Lotrič-Furlan S, Maraspin V (2002) Clinical and epidemiological findings for patients with erythema migrans. Comparisons of cohorts from the years 1993 and 2000. Wien klin wochenschr 114/13 $-14,493-497$.

25. Tylewska-Wierzbanowska S, Chmielewska T (2002) Limitation of serological testing for Lyme borreliosis: Evaluation of ELISA and Western blot in comparison with PCR and culture methods. Wien klin wochenschr 114/13 - 14: $601-605$.

26. Dattwyler RJ, Wormser GP, Rush TJ, Finkel MF, Schoen RT, Grunwaldt E et al (2005) A comparison of treatment regimens of ceftriaxone in late Lyme disease. Wien klin wochenschr 117 (11-12):393 - 397.

27. Berger B (1984) Erythema chronicum migrans of Lyme disease. Arch Dermatol 120:1017-1021.

28. Steere AC, Barthenhagen NH, Craft JE, Hutchinson GJ, Newman JH, Rahn DW, et al (1983) The early clinical manifestations of Lyme disease. Ann Int Med 99:76-82.

29. Wormser GP (2005) Prevention of Lyme borreliosis. Wien klin wochenschr 117 (11 12):385 - 391.

30. Bennet L, Halling A, Berglund J (2006) Increased incidence of Lyme borreliosis in southern Sweden following mild winters and during warm humid summers. Eur J Clin Microbiol Infect Dis 25(7):426-432.

31. Gray (2002) Biology of Ixodes species ticks in relation to tick - borne zoonoses. Wien klin wochenschr 114/13 - 14: 473 - 478.

32. Statistics $\quad$ Sweden. 1982-1999. (Data available from: http://www.scb.se/templates/Listning2_ 60938.asp). 
Table 1a. Characteristics of patients with erythema migrans infected with $B$. afzelii and $B$. garinii respectively.

\begin{tabular}{llll}
\hline Characteristic & $\begin{array}{l}\text { Patients } \\
\text { with B.afzelii } \\
(N=87)\end{array}$ & $\begin{array}{l}\text { Patients } \\
\text { with B. garinii } \\
(N=31)\end{array}$ & $p$ Value \\
\hline Male, $N(\%)$ & $47(54)$ & $17(54)$ & n.s. $^{2}$ \\
Age, median, years (range) & $55.0(19-78)$ & $61(30-94)$ & $0.005^{1}$
\end{tabular}

Size of lesion at presentation

median, $\mathrm{cm}^{2}$

$N$ (range)

Annular/Non-annular EM,

B. afzelii $N=58 ;$ B. garinii $N=19(\%)$

$34 / 24(58.6 / 41.4)$

Duration tick bite to diagnosis,

median, days

21.0

$N$ (range)

72 (3-97)

Duration from start of treatment

to disappearance of EM,

median, $\mathrm{d}$

8.0

$N$ (range)

$64(1-35)$

Treatment with

$\mathrm{pcV}, N(\%)$

doxycycline, $N(\%)$

$15(17.2)$

Geographic area at the time of the tick bite

coastal area, $N(\%) \quad 18(25.4)$

garden, $N(\%)$

$31(43.7)$

forest, $N(\%)$

$19(26.8)$
93.0

$30(24.0-414.0)$

n.s. ${ }^{1}$

$4 / 15(21.0 / 78.9)$

$0.004^{2}$

14.0

$24(4-78)$

$0.011^{1}$

7.0

$26(2-17)$

n.s. ${ }^{1}$

28 (90.3)

n.s ${ }^{2}$

3 ( 9.7)

$6(23.1)$

n.s $s^{3}$

13 (50.0)

4 (15.4)

${ }^{1}$ Mann-Whitney test, ${ }^{2}$ Two-sided Pearson chi square test, ${ }^{3}$ Kruskall-Wallis test. 
Table 1b. Clinical symptoms presenting in patients with erythema migrans caused by $B$. afzelii and B. garinii.

Clinical symptoms

Patients
with B.afzeli

$(N=87)$

$24(27.5)$

$12(13.8)$

$8(9.2)$

$6(6.9)$

Airway symptoms, $N(\%)$

Neurological symptoms, $N(\%)$

Fever, $N(\%)$

$0(0.0)$

Neckstifness, $N(\%)$

$3(3.4)$

$2(2.3)$

Photosensitivity, $N(\%)$

$8(9.2)$
Patients

$p$ Value $^{1}$

with B. garinii

$(N=31)$

$3(9.7)$

NS

$4(12.9)$

0.003

$1(3.2)$

NS

${ }^{1}$ Two-sided Pearson chi square test 
Table 1c. Laboratory findings in patients infected with B. afzelii and B. garinii, respectively.

Laboratory test

Patients

Patients

$p$ Value $^{1}$

with B.afzelii

with B. garinii

$(N=87)$

$(N=31)$

Positive serological results

$\begin{array}{lccc}\text { at presentation, } N(\%) & 24(35.8) & 12(54.5) & \text { n.s. } \\ \text { after } 6 \text { weeks, } N(\%) & 27(45.0) & 16(72.7) & 0.017 \\ \text { CRP }>8 \mathrm{mg} / \mathrm{L}, N(\%) & 13(14.9) & 12(38.7) & 0.006 \\ \text { WBC }<3.5 \times 10^{9} / \mathrm{L}, N(\%) & 2(2.3) & 0(0.0) & \text { NS } \\ \text { WBC }>8.8 \times 10^{9} / \mathrm{L}, N(\%) & 5(5.7) & 3(9.7) & \text { NS } \\ \text { Platelets }>350 \times 10^{9} / \mathrm{L}, N(\%) & 6(6.9) & 1(3.2) & \text { NS } \\ \text { Platelets }<150 \times 10^{9} / \mathrm{L}, N(\%) & 3(3.4) & 0(0.0) & \text { NS } \\ \text { ALT }>0.75 \mu \mathrm{kat} / \mathrm{L}(\mathrm{w}), N(\%) & & & \text { NS } \\ >1.10 \mu \mathrm{kat} / \mathrm{L}(\mathrm{m}), N(\%) & 9(10.3) & 3(9.7) & \\ \text { AST }>0.60 \mu \mathrm{kat} / \mathrm{L}(\mathrm{w}), N(\%) & & & \mathrm{NS} \\ >0.75 \mu \mathrm{kat} / \mathrm{L}(\mathrm{m}), N(\%) & 5(5.7) & 3(9.7) & \mathrm{NS}\end{array}$

${ }^{1}$ Two-sided Pearson chi square test

$\mathrm{CRP}=\mathrm{c}-$ reactive protein

$\mathrm{WBC}=$ white blood count

$\mathrm{ALT}=$ alanine aminotransferase

$\mathrm{AST}=$ aspartate aminotransferase

$\mathrm{LD}=$ lactate dehydrogenase

$\mathrm{w}=$ women

$\mathrm{m}=\mathrm{men}$ 
Table 2. Odds ratio of non-annular erythema migrans according to sex of patient and Borrelia genospecies. "Female sex" and "B. afzelii" are baseline variables. The odds ratio of the interaction factor between sex and genospecies is 12.46 (95\% CI: $0.98-158.80)$. N=77

\section{Sex of patient}

Female (95\% CI) $\mathrm{N}=26 \quad$ Male $(95 \% \mathrm{CI}) \mathrm{N}=13$

\section{Genospecies}

$\begin{array}{lll}\text { B. afzelii } & 1.00 & 0.09(0.03-0.33) \\ & (68 \%) & (16 \%) \\ \text { B. garinii } & 1.73(0.29-10.34) & 1.98(0.34-11.56) \\ & (78 \%) & (80 \%)\end{array}$

$\mathrm{CI}=$ Confidence interval 
Figure I. An example of an annular erythema migrans.

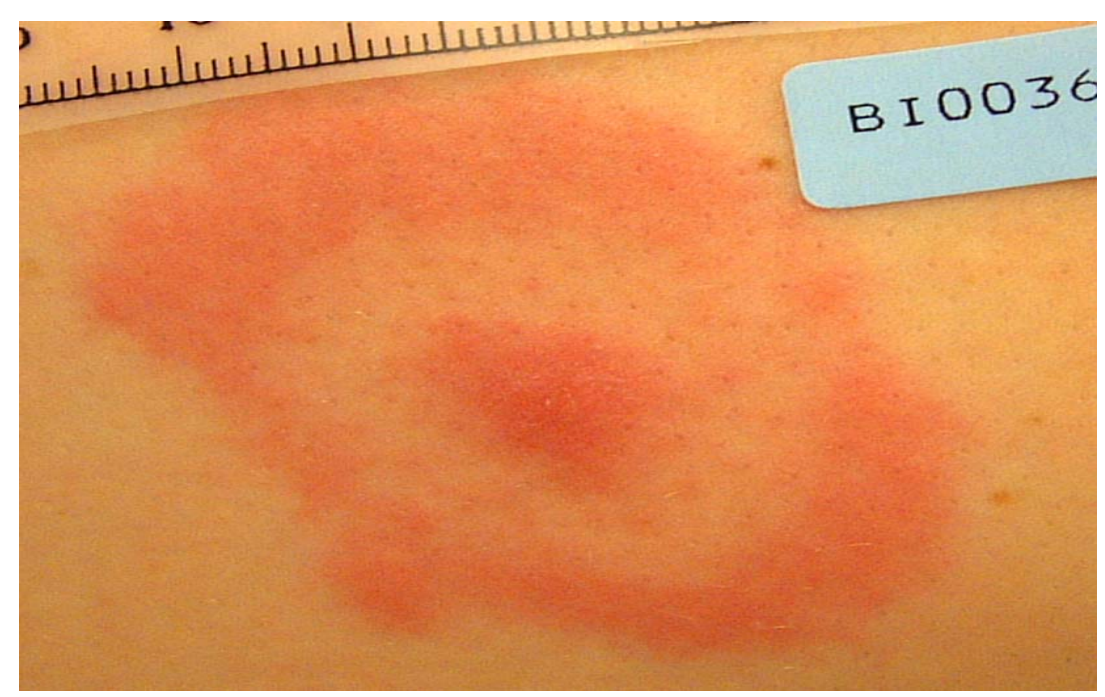


Figure II. An example of a non-annular erythema migrans.

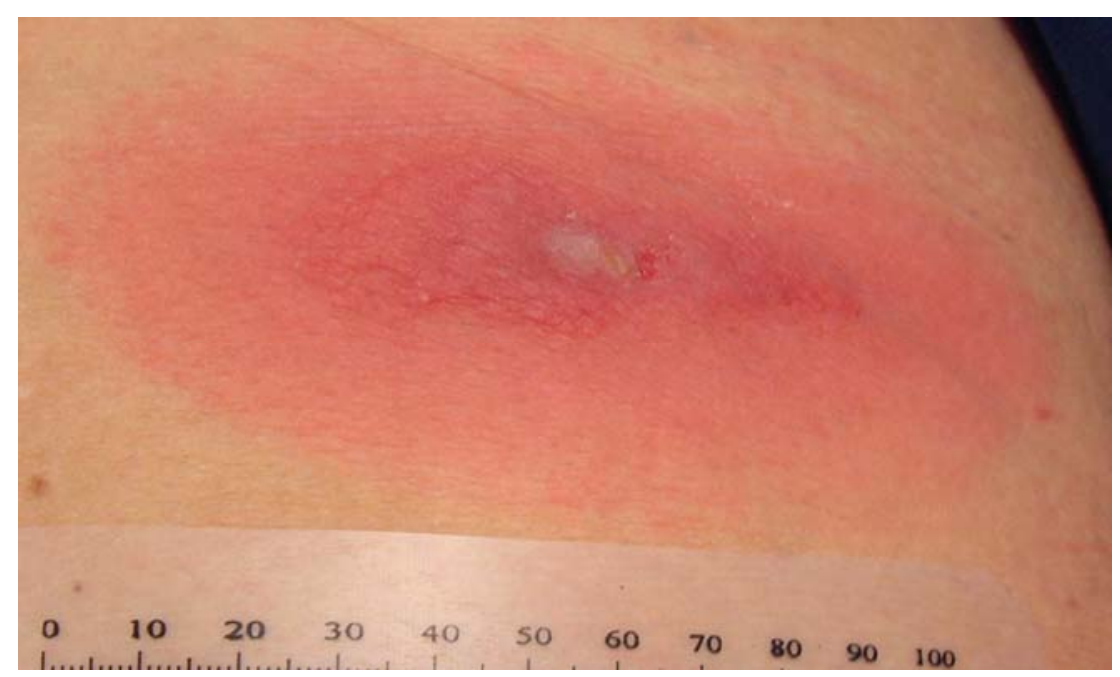


Figure III. The monthly distribution of tick bites for patients with erythema migrans caused by B. afzelii and B. garinii, respectively.

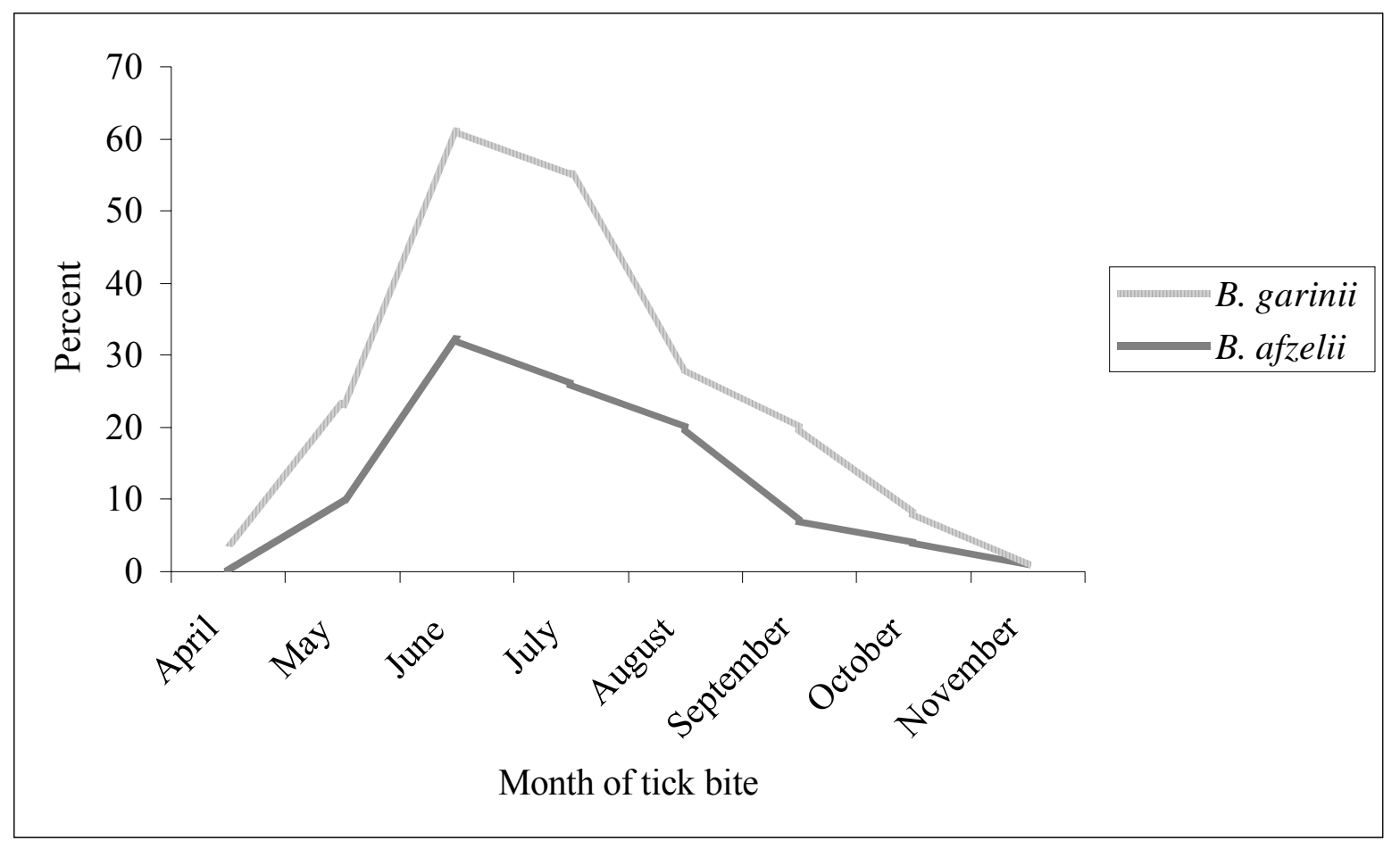

